\title{
Optical Lens Tinting-A Review of its Functional Mechanism, Efficacy, and Applications
}

\author{
Jared Raabe, ${ }^{1}$ Ashwini Kini, ${ }^{2}$ Andrew G Lee, ${ }^{2,3,4,5,5,6,7}$ \\ 1. University of Texas Medical Branch at Galveston, School of Medicine, Galveston, TX, USA; 2. Department of Ophthalmology, Blanton Eye Institute, \\ Houston Methodist Hospital, Houston, TX, USA; 3. Departments of Ophthalmology, Neurology, and Neurosurgery, Weill Cornell Medicine, New York, \\ NY, USA; 4. Department of Ophthalmology, University of Texas Medical Branch, Galveston, TX, USA; 5. University of Texas MD Anderson Cancer \\ Center, Houston, TX, USA; 6. Texas A\&M College of Medicine, Bryan, TX, USA; 7. Department of Ophthalmology, The University of Iowa Hospitals and \\ Clinics, Iowa City, IA, USA
}

DOI: https://doi.org/10.17925/USOR.2019.12.2.85

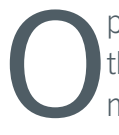
ptical filters are tinted lenses that preferentially filter light at a given wavelength. Filters that produce maximal filtration at $480 \mathrm{~nm}$ block the same wavelength that activates phototransduction in intrinsically photosensitive retinal ganglion cells, one pathway thought to mediate photosensitivity. Wavelength-specific optical filtration has shown promise as an adjunct treatment for a variety of medical conditions. This article will review the mechanism, efficacy, and various applications for wavelength-specific blockade, as well as discuss barriers to its implementation in clinical practice.

\section{Keywords}

FL-41, optical tinting, lens filter, melanopsin, photosensitivity, intrinsically photosensitive retinal ganglion cells

Disclosure: Jared Raabe, Ashwini Kini, and Andrew G Lee have nothing to declare in relation to this article. Review Process: Double-blind peer review.

Compliance with Ethics: This study involves a review of the literature and did not involve any studies with human or animal subjects performed by any of the authors.

Authorship: The named authors meet the International Committee of Medical Journal Editors (ICMJE) criteria for authorship of this manuscript, take responsibility for the integrity of the work as a whole, and have given final approval for the version to be published.

Received: August 3, 2019

Accepted: October 21, 2019

Citation: US Ophthalmic Review. 2019;12(2):85-7

Corresponding Author: Jared Raabe, University of Texas Medical Branch at Galveston, Schoo of Medicine, 301 University Blvd. Galveston,

TX 77555, USA. E: jmraabe@yahoo.com

Support: No funding was received in

the publication of this article.
Optical lens filtration therapy began with FL-41 lenses that were developed in the late 1980s and first reported in 1991 by Wilkins and Wilkinson. ${ }^{~}$ They were originally designed to improve workplace productivity by reducing eye strain and headaches induced by fluorescent lighting. They were designed to allow only $10 \%$ light filtration from $400-550 \mathrm{~nm}$ with a gradual increase in transmission from there with increased wavelength. ${ }^{1}$ The resulting lenses offered peak filtration at $480 \mathrm{~nm}$ and showed initial anecdotal efficacy in patients with agoraphobia, photosensitive epilepsy, post-traumatic photosensitivity, and eye strain. ${ }^{1}$ A subsequent study by Good et al., in the same year, showed a reduction in migraine frequency in children wearing the lenses. ${ }^{2}$ This initial design occurred many years before the discovery of intrinsically photosensitive retinal ganglion cells (IPRGCS) and its relation to the pathophysiology of photosensitivity. ${ }^{3,4}$ This article will discuss the mechanism and efficacy for various applications of optical lens filters and wavelength-specific blockade.

The literature was reviewed with PubMed queries for "FL-41", "Iens filters photosensitivity", "lens filters photophobia", "axon optics photophobia", and "lens filters melanopsin". Articles were identified from the year 1990 to the present that discussed the pathophysiology of photophobia and optical filtration therapy. References from included articles were also examined in search of other pertinent articles.

\section{Mechanism}

Blue-blocking lenses, such as the original FL-41, preferentially block shorter wavelengths of light in the visible spectrum, usually including significant filtration around $480 \mathrm{~nm} .{ }^{1,5}$ This appears to be a particularly important wavelength, because it is also the wavelength of light that induces phototransduction in IPRGCS, which were discovered around a decade later. ${ }^{3,4}$ These retinal ganglion cells are labeled "intrinsically photosensitive" because they are able to transduce light signals independent of traditional rod or cone photoreceptors., ${ }^{3,6-10}$ They have been shown to have involvement in both circadian rhythms and the pupillary light reflex. ${ }^{3.4}$ Modulation of IPRGC phototransduction is thought to be influenced by melanopsin, a biphasic pigment found in a IPRGCs that isomerizes between its all-trans and 11-cis forms upon exposure to $481 \mathrm{~nm}$ and $587 \mathrm{~nm}$ light, respectively. This means that the original blue-blocking lenses were unknowingly developed with a peak filtration that minimized the isomerization of melanopsin, and therefore, reduced rod-cone independent phototransduction by IPRGCS.

Multiple pathways have been theorized to mediate photophobia (e.g., trigeminal, sympathetic mediation), ${ }^{6,10,11}$ but one of those is directly mediated by IPRGCS, with signal transduction to 
nociceptive centers in the thalamus, ${ }^{6,8}$ an area that has shown positron emission tomography activation in photophobic patients with essential blepharospasm. ${ }^{12}$ This concurs with pre-existing evidence from Stringham et al. that shows that the symptoms of photophobia may increase with exposure to shorter wavelengths of light,13 and newer evidence from Zivcevska et al. that also showed greater sensitivity to light that corresponded to melanopsin's peak spectral sensitivity in both healthy and clinical patients. ${ }^{14}$ The study by Stringham et al. was in three healthy subjects, and though it showed increased photosensitivity with shorter wavelengths, their action spectrum peaked around $520 \mathrm{~nm}$, later than the melanopsin absorption spectrum. ${ }^{13}$ Additionally, a recent study by Marek et al. showed alleviation of blue-light (shorter wavelength) mediated photosensitivity in mice through application of a melanopsin antagonist. ${ }^{9}$

Therefore, theoretically, lenses that reduce exposure to light around $481 \mathrm{~nm}$ would decrease isomerization of melanopsin to its all-trans form and subsequent IPRGC-mediated phototransduction to nociceptive centers in the posterior thalamus. This is one proposed mechanism for the use of blue-blocking lenses as an adjunct treatment to a variety of conditions that champion photosensitivity as a prominent symptom. Other proposed mechanisms include increased comfort due to a reduction in overall light, sensitivity to a wavelength other than $480 \mathrm{~nm}$, or the presence of a placebo effect when wearing tinted lenses.

\section{Applications}

Historical applications of tinted lenses have included treatment of patients with migraines, traumatic brain injury, benign essential blepharospasm (BEB), sleep difficulties, post-traumatic photosensitivity, and retinal dystrophies. ${ }^{2,5,9,15-23}$ In theory, however, they could be therapeutic in any patient with a photophobia-inducing condition, though they may be more effective in conditions whose nociceptive component relies more heavily on an IPRGC-mediated neural pathway. Various pathologies that may induce photophobia can be ocular (e.g., dry eye, uveitis, cone dystrophy), neurologic (e.g., optic neuritis, demyelinating disorders, migraine), or medication-related (e.g., lithium, benzodiazepines, chloroquine) in origin, among many other ailments (e.g., intracranial neoplasm, zinc deficiency).$^{6.10}$ Additionally, the notion that optical filters may have clinical potential in the treatment of photophobia is supported by many patients experiencing photophobic symptoms after cataract surgery. The appearance of photophobic symptoms after the removal of a patient's intrinsic light filter may suggest that a filter could be added to mitigate these same symptoms.

\section{Migraine}

Migraines were one of the first diseases targeted by optic-lens tinting due to their prominent photophobia. In 1991, Good et al. randomized 20 children with clinically diagnosed migraines to one cohort wearing blue-tinted and another wearing rose-tinted glasses. Only the patients wearing rose-tinted glasses (blue-light blocking) reported symptom reduction. ${ }^{2}$ A few years later, Main et al. reported that patients had a lower discomfort threshold to low wavelength light between migraine attacks (compared with medium and high wavelength). ${ }^{16}$ This has been reinforced with functional magnetic resonance imaging (fMRI) data that showed decreased cortical activation and subjective decreases in illusions and distortions in patients with migraine who wore precision optical tints when exposed to stressful striped images. ${ }^{24}$ To more precisely test this hypothesis, Hoggan et al. produced an optical notch filter (named for the shape of its light transmission curve) that more specifically blocks $480 \mathrm{~nm}$ light. This serves the same purpose as the traditional FL-41 tint, but it uses thin film technology instead of directly dying the lens to provide a more precise spectral filter. It was tested in patients with migraine, and symptoms were relieved with both a $480 \mathrm{~nm}$ and $620 \mathrm{~nm}$ filter. ${ }^{22}$ This further suggests that wavelength-specific blockade is likely a viable treatment option for patients migraine; however, it also presents the possibility that the improved comfort could be due to an overall reduction of light. The efficacy seen with $620 \mathrm{~nm}$ blockade is hypothesized to relate to the bi-stable nature of melanopsin and merits further investigation. ${ }^{22}$

\section{Traumatic brain injury}

Optical tinting has also shown promise in the treatment of patients with traumatic brain injury, who commonly suffer from chronic photophobia as a component of post-concussion syndrome..$^{25}$ In 1996, a cohort of patients with traumatic brain injury was shown to have improved binocular letter contrast sensitivity when looking through Corning photochromic filters. ${ }^{15}$ More recently, a small study was performed with 12 participants who had all suffered a mild traumatic brain injury and now complained of persistent reading difficulties and photophobia. Though all of the patients reported a greater degree of comfort with tinted lenses, there were no significant differences in objective reading parameters. ${ }^{21}$ Though limited by sample size, this suggests that optical tinting will not likely supplant vision rehabilitation as the primary treatment for post-concussion syndrome. It does still show promise, however, as an adjunct treatment, as all of the patients in the cohort reported increased subjective comfort. ${ }^{21}$

\section{Benign essential blepharospasm}

BEB has been a focus of several studies hoping to provide relief to photophobic patients via optical filtration. It is characterized by focal, dystonic, involuntary contractions of eyelid protractors. ${ }^{5}$ Since the pathophysiology of BEB is incompletely understood, treatment is generally focused on symptomatic relief (e.g., botulinum toxin injections, facial nerve avulsion, eyelid protractor myomectomy). Up to $79 \%$ of patients with BEB report photophobia as a prominent symptom, as it can both induce spasm and continue to be uncomfortable between spasms. ${ }^{5}$ In 2005, Herz and Yen performed a non-randomized case-control study of 34 patients (24 BEB patients and 10 controls) who were all subjected to a light source of increasing intensity. This was repeated with seven different optical filters. Though a different filter allowed patients to tolerate the highest intensity of light, 17 of the 24 patients with BEB preferred the FL-41 lens, with no other filter receiving more than two votes. ${ }^{5} \mathrm{~A}$ year later, another similar study was performed by Adams et al. with patients with BEB that reported impairment in activities of daily living related to photophobia. FL-41 tinted lenses showed improvement in this cohort as well; however, they were not shown to be superior to grey (neutrally) tinted lenses as these also significantly alleviated symptoms. ${ }^{18}$

In 2009, the hypothesis that FL-41 lens tinting might help patients with BEB was tested by Blackburn et al., who performed two studies reported in the same paper.20 One study had patients wear either FL-41 tinted lenses or grey tinted lenses for 2 weeks and then the other lens for another 2 weeks after a 2-week washout period. Patients were given questionnaires at baseline and after wearing each lens. The other study used surface electromyography to measure blink frequency, duration, and force while wearing FL-41, rose-, or grey-tinted lenses. Though improvement was seen with both FL-41 and grey lenses, FL-41 tinting led to more improvement in 
the metrics for reading, fluorescent light sensitivity, overall light sensitivity, blepharospasm frequency, and blepharospasm severity. They also reduced mean blink rate more than grey or rose-tinted lenses. ${ }^{20}$

Collectively, these findings demonstrate the FL-41 tinted lenses are likely a useful treatment option for patients suffering from photophobia secondary to BEB. Though patients may benefit from either FL-41 or grey tint, there is some evidence to suggest that $\mathrm{FL}-41$ might be superior. These findings may also extend to other blue-blocking lenses that target a similar spectrum. We argue that tinted lenses are certainly worth a trial before considering more invasive treatments for BEB.

\section{Retinal disorders}

We found two studies that evaluated the use of optical tinting in patients with retinal cone disorders. 17,19 In 2004, Park and Sunness fit 24 patients with either achromatopsia or an acquired cone disorder who complained of severe photophobia with soft red contact lenses with hopes of symptom alleviation. It immediately reduced light-aversion in all patients. Eight patients became eligible to drive in Michigan or Maryland (defined as 20/100 or better at the time of the study) and all became full-time wearers. The lenses used transmitted 30\% from 400-480 nm and not at all in the middle of the visible spectrum. ${ }^{17}$

The second study was performed by Rajak etal. in 2006, fitting three patients with cone dystrophies who all had markedly decreased visual acuity and severe photophobia with Lunelle ES70 Solaire 70\% brown contact lenses. ${ }^{19}$ The spectral transmission of the lenses was not described. Two of the three children and their parents reported subjective improvement in confidence, peer-to-peer interaction, and a decrease in bullying which was born out objectively for one child upon evaluation with a Children's Visual Function questionnaire. The third child was reluctant to wear the lenses.

Collectively, these two studies demonstrate great promise for optical tinting as a possible treatment for patients with retinal disorders suffering from photophobia. Further studies with larger sample sizes and testing with lenses of different spectral transmission would be intriguing due to the reported efficacy in these studies and the non-invasive nature of the treatment.

\section{Sleep difficulty and other psychiatric conditions}

Though photophobia has been reported in patients with attention deficit hyperactivity disorder (ADHD), panic disorder, ${ }^{26,27}$ agoraphobia, ${ }^{26}$ anxiety, and depression, ${ }^{6}$ it is usually accompanied by additional pathology that may be contributing to the photophobia. ${ }^{6}$ Patients with agoraphobia have shown symptomatic improvement in photophobia after cognitive behavioral therapy. ${ }^{26}$ This suggests that their photophobia is likely related to their psychiatric illness as opposed to an external process. Though we found no studies that evaluated optical tinting in patients with psychiatric illness, the presence of photophobia in this population suggests that they may also benefit. Further studies are needed to provide evidence.

\section{Barriers to implementation}

Though much evidence exists suggesting that FL-41 or other optical tinting is likely a useful treatment for patients with photophobia from a variety of etiologies, it is often not implemented. Barriers to clinical use in Canada were described by Lee et al. ${ }^{28}$ to be related to availability. Out of 88 optical shops that responded (192 were asked) in Toronto and Vancouver, only seven reported offering tinted lens services. The shops cited lack of awareness of FL-41 and its indications, low customer demand, and lack of necessary equipment as barriers. The lenses are available online, but customers may be subject to higher prices, inability to test products, and a lack of information specific to their unique needs. ${ }^{28}$ Katz and Digre also report low availability of tinted optics to be a problem in the United States. ${ }^{6}$

\section{Conclusion}

some reports suggest that blue-blocking or optic notch lenses reducing transmission around $480 \mathrm{~nm}$ can provide a significant benefit to patients suffering from photophobia of a variety of etiologies. This appears to be due to blockade of light that triggers the isomerization of melanopsin, inducing phototransduction in IPRGCs leading to activation of nociceptive centers in the thalamus. More studies are needed to explore wavelengthspecific filtration as a potential treatment for a several different conditions. More practitioners should consider implementation of tinted optics in their clinical practice, hopefully leading to a rise in availability. We believe many patients stand to benefit from wavelength-specific light filtration in the future. $\square$
1. Wilkins AJ, Wilkinson P. A tint to reduce eye-strain from fluorescent lighting? Preliminary observations. Ophthalmic Physiol Opt. 1991;11:172-5

2. Good PA, Taylor RH, Mortimer MJ. The use of tinted glasses in childhood migraine. Headache. 1991;31:533-6.

3. Berson DM, Dunn FA, Takao M. Phototransduction by retinal ganglion cells that set the circadian clock. Science. 2002:295:1070-3.

4. Hattar S, Liao HW, Takao M, et al. Melanopsin-containing retinal ganglion cells: architecture, projections, and intrinsic photosensitivity. Science. 2002;295:1065-70.

5. Herz NL, Yen MT. Modulation of sensory photophobia in essential blepharospasm with chromatic lenses. Ophthalmology. 2005;112:2208-11.

6. Katz BJ, Digre KB. Diagnosis, pathophysiology, and treatment of photophobia. Surv Ophthalmol. 2016;61:466-77.

7. Mure LS, Cornut PL, Rieux C, et al. Melanopsin bistability: a fly's eye technology in the human retina. PLoS One. 2009;4:e5991.

8. Noseda R, Kainz V, Jakubowski M, et al. A neural mechanism for exacerbation of headache by light. Nat Neurosci. 2010;13:239-45.

9. Marek V, Reboussin E, Dégardin-Chicaud J, et al. Implication of melanopsin and trigeminal neural pathways in blue light photosensitivity in vivo. Front Neurosci. 2019;13:497.

10. Digre KB, Brennan KC. Shedding light on photophobia. I Neuro-Ophthalmology. 2012;32:68-81.

11. Moulton EA, Becerra L, Borsook D. An fMRI case report of photophobia: activation of the trigeminal nociceptive pathway. Pain. 2009;145:358-63.

12. Emoto $H$, Suzuki $Y$, Wakakura $M$, et al. Photophobia in essential blepharospasm-A positron emission tomographic study. Mov Disord. 2010;25:433-9.

13. Stringham JM, Fuld $K$, Wenzel AJ. Action spectrum for photophobia. J Opt Soc Am A Opt Image Sci Vis. 2003;20:1852-8.

14. Zivcevska M, Lei S, Blakeman A, et al. A novel visual psychometric test for light-induced discomfort using red and blue light stimuli under binocular and monocular viewing conditions. Investig Opthalmology Vis Sci. 2018;59:1467.

15. Jackowski MM, Sturr JF, Taub HA, Turk MA. Photophobia in patients with traumatic brain injury: uses of light-filtering lenses to enhance contrast sensitivity and reading rate. NeuroRehabilitation. 1996;6:193-201.

16. Main A, Vlachonikolis I, Dowson $A$. The wavelength of light causing photophobia in migraine and tension-type headache between attacks. Headache. 2000;40:194-9.

17. Park WL, Sunness JS. Red contact lenses for alleviation of photophobia in patients with cone disorders. Am J Ophthalmol. 2004;137:774-5.

18. Adams WH, Digre KB, Patel BCK, et al. The evaluation of ligh sensitivity in benign essential blepharospasm. Am J Ophthalmol. 2006:142:82-7.e8.

19. Rajak SN, Currie ADM, Dubois VJP, et al. Tinted contact lenses as an alternative management for photophobia in stationary cone dystrophies in children. J Am Assoc Pediatr Ophthalmo Strabismus. 2006;10:336-9.

20. Blackburn MK, Lamb RD, Digre KB, et al. FL-41 tint improves blink frequency, light sensitivity, and functional limitations in patients with benign essential blepharospasm. Ophthalmology. 2009:116:997-1001.

21. Fimreite $\mathrm{V}$, Willeford KT, Ciuffreda KJ. Effect of chromatic filters on visual performance in individuals with mild traumatic brain injury (mTBI): a pilot study. J Optom. 2016;9:231-9.

22. Hoggan RN, Subhash A, Blair S, et al. Thin-film optical notch filter spectacle coatings for the treatment of migraine and photophobia. J Clin Neurosci. 2016;28:71-6.

23. Main A, Dowson A, Gross M. Photophobia and phonophobia in migraineurs between attacks. Headache. 1997;37:492-5.

24. Huang J, Zong $X$, Wilkins $A$, et al. fMRI evidence that precision ophthalmic tints reduce cortical hyperactivation in migraine. Cephalalgia. 2011;31:925-36.

25. Armstrong RA. Visual problems associated with traumatic brain injury. Clin Exp Optom. 2018;101:716-26.

26. Kellner M, Wiedemann K, Zihl J. Illumination perception in photophobic patients suffering from panic disorder with agoraphobia. Acta Psychiatr Scand. 1997;96:72-4.

27. Bossini L, Fagiolini $A$, Valdagno $M$, et al. Photosensitivity in panic disorder. Depress Anxiety. 2009;26:E34-6.

28. Lee EY, Emami S, Cho R, Ing E. Availability of FL-41 lens tint in Toronto and Vancouver. Can J Ophthalmol. 2018;53:e169-70 